\title{
On a model for electromagnetic processes inside and outside a ferromagnetic body
}

\author{
Martin Brokate ${ }^{1}$, Michela Eleuteri ${ }^{2}$ and Pavel Krejčí ${ }^{3}$
}

\begin{abstract}
One-dimensional Maxwell's equations are considered in a ferromagnetic body surrounded by vacuum. Existence and uniqueness of solution for the resulting system of P.D.E.s with hysteresis on the whole real line is proved under suitable constitutive hypotheses.
\end{abstract}

Classification: 35Q60, 47J40.

Key words: electromagnetic processes, ferromagnetic materials, hysteresis.

\section{Introduction}

The aim of this paper is to find existence and uniqueness of a solution for the following model system

$$
\begin{cases}\frac{\partial E}{\partial t}+\chi_{\Omega}\left(E+E_{\mathrm{app}}\right)+\left(1-\chi_{\Omega}\right) J_{\mathrm{ext}}+\frac{\partial H}{\partial x}=0 & \\ \frac{\partial B}{\partial t}+\frac{\partial E}{\partial x}=0 & \text { in } \mathbb{R} \times(0, T) \\ H=\chi_{\Omega}\left(\overline{\mathcal{G}}(B)+\gamma \frac{\partial B}{\partial t}\right)+\left(1-\chi_{\Omega}\right) B & \end{cases}
$$

where $\Omega$ is an open bounded interval of the real line, $\chi_{\Omega}$ is the characteristic function of the set $\Omega, \overline{\mathcal{G}}$ is a suitable scalar hysteresis operator, $\gamma$ is a given positive constant while $E_{\text {app }}$ and $J_{\text {ext }}$ are known functions.

This system arises in the context of electromagnetic processes. We show in the next section how it can be obtained by coupling in a suitable way the Maxwell equations, the Ohm law and a constitutive relation between the magnetic field and the magnetic induction.

A similar equation was considered in [5] in the case of prescribed boundary conditions on $\partial \Omega$. Here we consider instead the evolution in the whole space, replacing the boundary conditions by the continuity of $E$ and $H$ across $\partial \Omega$.

\footnotetext{
${ }^{1}$ Zentrum Mathematik (M6), TU München, Boltzmannstr. 3, D-85747 Garching b. München, Germany. E-mail: brokate@ma.tum.de.

${ }^{2}$ Weierstrass-Institute for Applied Analysis and Stochastics, Mohrenstr. 39, D-10117 Berlin, Germany. E-mail: eleuteri@wias-berlin.de. Corresponding author.

${ }^{3}$ Weierstrass-Institute for Applied Analysis and Stochastics, Mohrenstr. 39, D-10117 Berlin, Germany. E-mail: krejci@wias-berlin.de. On leave from the Mathematical Institute, Academy of Sciences of the Czech Republic, Žitná 25, CZ-11567 Praha 1.
} 
Some details of the physical motivation for (1.1) are contained in Section 2; in Section 3 we recall some basic fact about hysteresis and hysteresis operators; Section 4 contains the statement of the main results which will be proved finally in Sections 5 and 6 .

\section{Physical motivation}

Consider an electromagnetic process in a ferromagnetic material which occupies a Euclidean domain $Q \subset \mathbb{R}^{3}$ in a time interval $(0, T)$. For more details on these topics we refer for example to [6]. From now on we set

$$
Q_{T}:=Q \times(0, T) \quad \mathbb{R}_{T}^{3}:=\mathbb{R}^{3} \times(0, T) .
$$

We suppose for simplicity that the electric displacement $\vec{D}$ is proportional to the electric field $\vec{E}$, that is $\vec{D}=\epsilon \vec{E}$, where $\epsilon$ is the electric permittivity. We assume that $\epsilon$ is a scalar constant and moreover we introduce the electric conductivity $\sigma$ which is supposed to vanish outside $Q$. We denote by $\vec{E}_{\text {app }}$ a prescribed applied electromotive force; then the Ohm law for the electric current $\vec{J}$ is given by the following relation

$$
\vec{J}= \begin{cases}\sigma\left(\vec{E}+\vec{E}_{\mathrm{app}}\right) & \text { in } Q \times(0, T) \\ \vec{J}_{\mathrm{ext}} & \text { in }\left[\mathbb{R}^{3} \backslash Q\right] \times(0, T) .\end{cases}
$$

Using the characteristic function $\chi_{Q}$ of the set $Q$, i.e. $\chi_{Q}=1$ inside $Q$ and $\chi_{Q}=0$ outside $Q$, we rewrite $(2.1)$ as

$$
\vec{J}=\chi_{Q} \sigma\left(\vec{E}+\vec{E}_{\mathrm{app}}\right)+\left(1-\chi_{Q}\right) \vec{J}_{\mathrm{ext}} \quad \text { in } \mathbb{R}_{T}^{3} .
$$

Now we recall the Ampère and the Faraday laws

$$
\begin{array}{ll}
c \nabla \times \vec{H}=4 \pi \vec{J}+\epsilon \frac{\partial \vec{E}}{\partial t} & \text { in } \mathbb{R}_{T}^{3} \\
c \nabla \times \vec{E}=-\frac{\partial \vec{B}}{\partial t} & \text { in } \mathbb{R}_{T}^{3}
\end{array}
$$

where $\vec{H}$ is the magnetic field, $\vec{B}$ is the magnetic induction and $c$ is the speed of light in vacuum.

Our analysis does not depend on the exact value of the constants $\sigma, c, \epsilon, \pi$. In order to simplify the presentation, we therefore consider the system

$$
\left\{\begin{array}{ll}
\nabla \times \vec{H} & =\chi_{Q}\left(\vec{E}+\vec{E}_{\mathrm{app}}\right)+\left(1-\chi_{Q}\right) \vec{J}_{\mathrm{ext}}+\frac{\partial \vec{E}}{\partial t} \\
\nabla \times \vec{E} & =-\frac{\partial \vec{B}}{\partial t}
\end{array} \quad \text { in } \mathbb{R}_{T}^{3} .\right.
$$

We further simplify our model system (2.5) by dealing with planar waves only. More precisely, we consider an electromagnetic wave moving in a plane which we suppose for 
simplicity to be orthogonal to the $x$-axis, in a domain $Q=\{(x, y, z): x \in \Omega,(y, z) \in$ $\left.\mathbb{R}^{2}\right\}$, with $\Omega$ being a bounded interval of the real line.

We assume that $\vec{E}$ is parallel to the $y$-axis, i.e.

$$
\vec{E}(x, t)=(0, E(x, t), 0) \quad(x, t) \in \mathbb{R} \times(0, T) .
$$

This in turn implies the following restriction on $\vec{B}$

$$
\vec{B}(x, t)=(0,0, B(x, t)) \quad(x, t) \in \mathbb{R} \times(0, T)
$$

and therefore also $\vec{H}(x, t)=(0,0, H(x, t))$ for all $(x, t) \in \mathbb{R} \times(0, T)$. Thus we have

$$
\nabla \times \vec{H}=\left(0,-\frac{\partial H}{\partial x}, 0\right) \quad \nabla \times \vec{E}=\left(0,0, \frac{\partial E}{\partial x}\right)
$$

and (2.5) reduces to a one-dimensional problem

$$
\begin{cases}\frac{\partial E}{\partial t}+\chi_{\Omega}\left(E+E_{\mathrm{app}}\right)+\left(1-\chi_{\Omega}\right) J_{\mathrm{ext}}+\frac{\partial H}{\partial x}=0 & \text { in } \mathbb{R} \times(0, T) \\ \frac{\partial B}{\partial t}+\frac{\partial E}{\partial x}=0 & \end{cases}
$$

where we also set $\vec{E}_{\text {app }}(x, t)=\left(0, E_{\text {app }}(x, t), 0\right)$ and $\vec{J}_{\text {ext }}(x, t)=\left(0, J_{\text {ext }}(x, t), 0\right)$.

We couple this equation with an appropriate constitutive relation. We choose to relate $B$ and $H$ by means of a constitutive law with hysteresis inside $\Omega$ and to set $B=H$ outside $\Omega$.

The constitutive law between $B$ and $H$ inside $\Omega$ will be chosen according to the "rheological" circuit model $F-L$ as in [19, p. 54-55], where a ferromagnetic element

$$
F: B^{F}=H^{F}+4 \pi M=(I+\overline{\mathcal{W}})\left(H^{F}\right)
$$

where $M$ is the magnetization and $\overline{\mathcal{W}}$ is a scalar Preisach operator, is coupled in series with an induction element

$$
L: H^{L}=\gamma \frac{\partial B^{L}}{\partial t} .
$$

The general rheological rule for series combinations yields

$$
B:=B^{F}=B^{L} \quad H:=H^{F}+H^{L},
$$

where $B$ is the total induction and $H$ is the total field. Summing up we obtain

$$
H=\chi_{\Omega}\left(\overline{\mathcal{G}}(B)+\gamma \frac{\partial B}{\partial t}\right)+\left(1-\chi_{\Omega}\right) B
$$

where we set $\overline{\mathcal{G}}:=(I+\overline{\mathcal{W}})^{-1}$. By coupling (2.6) and (2.7) we finally obtain (1.1). 


\section{Hysteresis operators}

The theory of hysteresis has a long history. A hundred years ago, Madelung in [15] proposed probably the first axiomatic approach to hysteresis by defining three experimental laws of what we call nowadays return point memory hysteresis (or "wiping-out property", cf. [16]). The model for ferromagnetic hysteresis proposed by Preisach in 1935 in [17] is a prominent representative that possesses the return point memory property. Only recently, Brokate and Sprekels proved (see [2, Theorem 2.7.7]) that every return point memory hysteresis operator, which admits a specific initial memory configuration, has necessarily a Preisach-type memory structure. A basic mathematical theory of hysteresis operators has been developed by Krasnosel'skil and his collaborators. The results of this group are summarized in the monograph [9], which constitutes until now the main source of reference on hysteresis. Our presentation here is based on more recent results from $[11,12]$ that are needed here, in particular the alternative one-parametric formulation of the Preisach model based on variational inequalities.

\subsection{The Preisach operator}

We describe the ferromagnetic behaviour using the Preisach model (see [17]). Mathematical aspects of this model were investigated by Krasnosel'skiı and Pokrovskiur (see [7], [8], and [9]). The model has been also studied in connection with partial differential equations by Visintin (see for example [18], [19]). The monograph of Mayergoyz ([16]) is mainly devoted to its modeling aspects.

Here we use the one-parametric representation of the Preisach operator which goes back to [10]. The starting point of our theory is the so-called play operator. This operator constitutes the simplest example of continuous hysteresis operator in the space of continuous functions; it has been introduced in [9] but we can also find equivalent definitions in [2] and [19]; for its extension to less regular inputs, see also [13] and [14]. Let $r>0$ be a given parameter. For a given input function $u \in \mathcal{C}^{0}([0, T])$ and initial condition $x^{0} \in[-r, r]$, we define the output $\xi=\mathcal{P}_{r}\left(x^{0}, u\right) \in \mathcal{C}^{0}([0, T]) \cap B V(0, T)$ of the play operator

$$
\mathcal{P}_{r}:[-r, r] \times \mathcal{C}^{0}([0, T]) \rightarrow \mathcal{C}^{0}([0, T]) \cap B V(0, T)
$$

as the solution of the variational inequality in Stieltjes integral form

$$
\begin{cases}\int_{0}^{T}(u(t)-\xi(t)-y(t)) d \xi(t) \geq 0 & \forall y \in \mathcal{C}^{0}([0, T]), \quad \max _{0 \leq t \leq T}|y(t)| \leq r \\ |u(t)-\xi(t)| \leq r & \forall t \in[0, T], \\ \xi(0)=u(0)-x^{0} & \end{cases}
$$

Let us consider now the whole family of play operators $\mathcal{P}_{r}$ parameterized by $r>$ 0 , which can be interpreted as a memory variable. Accordingly, we introduce the hysteresis memory state space

$$
\Lambda:=\left\{\lambda: \mathbb{R}_{+} \rightarrow \mathbb{R}:|\lambda(r)-\lambda(s)| \leq|r-s| \forall r, s \in \mathbb{R}_{+}: \lim _{r \rightarrow+\infty} \lambda(r)=0\right\},
$$


together with its subspaces

$$
\Lambda_{\tilde{R}}=\{\lambda \in \Lambda: \lambda(r)=0 \text { for } r \geq \tilde{R}\}, \quad \Lambda_{\infty}=\bigcup_{\tilde{R}>0} \Lambda_{\tilde{R}} .
$$

For $\lambda \in \Lambda, u \in \mathcal{C}^{0}([0, T])$ and $r>0$ we set

$$
\wp_{r}[\lambda, u]:=\mathcal{P}_{r}\left(x_{r}^{0}, u\right) \quad \wp_{0}[\lambda, u]:=u,
$$

where $x_{r}^{0}$ is given by the formula

$$
x_{r}^{0}:=\min \{r, \max \{-r, u(0)-\lambda(r)\}\} .
$$

We set

$$
A(r, u(0)):=u(0)-x_{r}^{0}
$$

It turns out that

$$
\wp_{r}: \Lambda \times \mathcal{C}^{0}([0, T]) \rightarrow \mathcal{C}^{0}([0, T])
$$

is Lipschitz continuous in the sense that, for every $u, v \in \mathcal{C}^{0}([0, T]), \lambda, \mu \in \Lambda$ and $r>0$ we have

$$
\left\|\wp_{r}[\lambda, u]-\wp_{r}[\mu, v]\right\|_{\mathcal{C}^{0}([0, T])} \leq \max \left\{|\lambda(r)-\mu(r)|,\|u-v\|_{\mathcal{C}^{0}([0, T])}\right\}
$$

Moreover, if $\lambda \in \Lambda_{R}$ and $\|u\|_{\mathcal{C}^{0}([0, T])} \leq R$, then $\wp_{r}[\lambda, u](t)=0$ for all $r \geq R$ and $t \in[0, T]$. For more details, see Sections II.3, II.4 of [11].

Now we introduce the Preisach plane as follows

$$
\mathscr{P}:=\left\{(r, v) \in \mathbb{R}^{2}: r>0\right\}
$$

and consider a function $\varphi \in L_{\mathrm{loc}}^{1}(\mathscr{P})$ such that there exists $\beta_{1} \in L_{\mathrm{loc}}^{1}(0, \infty)$ with

$$
0 \leq \varphi(r, v) \leq \beta_{1}(r) \quad \text { for a.e. }(r, v) \in \mathscr{P} .
$$

We set

$$
g(r, v):=\int_{0}^{v} \varphi(r, z) d z \quad \text { for }(r, v) \in \mathscr{P}
$$

and for $R>0$, we put $b_{1}(R):=\int_{0}^{R} \beta_{1}(r) d r$.

Then the Preisach operator

$$
\mathcal{W}: \Lambda_{\infty} \times \mathcal{C}^{0}([0, T]) \rightarrow \mathcal{C}^{0}([0, T])
$$

generated by the function $g$ is defined by the formula

$$
\mathcal{W}[\lambda, u](t):=\int_{0}^{\infty} g\left(r, \wp_{r}[\lambda, u](t)\right) d r
$$

for any given $\lambda \in \Lambda_{\infty}, u \in \mathcal{C}^{0}([0, T])$ and $t \in[0, T]$. The equivalence of this definition and the classical one in [16], [19], e.g., is proved in [10]. 
The function $A(r, \cdot)$ in $(3.3)$ is nondecreasing and Lipschitz continuous. Hence, the mapping

$$
\mathscr{A}: \mathbb{R} \rightarrow \mathbb{R}: \quad u(0) \mapsto \int_{0}^{\infty} g(r, A(r, u(0))) d r
$$

which with the initial input value $u(0)$ associates the initial output value $\mathcal{W}[\lambda, u](0)$ is nondecreasing and Lipschitz continuous as well.

Using the Lipschitz continuity (3.4) of the operator $\wp_{r}$, it is easy to prove that also $\mathcal{W}$ is locally Lipschitz continuous, in the sense that, for any given $R>0$, for every $\lambda, \mu \in \Lambda_{R}$ and $u, v \in \mathcal{C}^{0}([0, T])$ with $\|u\|_{\mathcal{C}^{0}([0, T])},\|v\|_{\mathcal{C}^{0}([0, T])} \leq R$, we have

$$
\|\mathcal{W}[\lambda, u]-\mathcal{W}[\mu, v]\|_{\mathcal{C}^{0}([0, T])} \leq \int_{0}^{R}|\lambda(r)-\mu(r)| \beta_{1}(r) d r+b_{1}(R)\|u-v\|_{\mathcal{C}^{0}([0, T])} .
$$

The first result on the inverse Preisach operator was proved in [3]. We make use of the following formulation proved in [11], Section II.3.

Theorem 3.1. Let $\lambda \in \Lambda_{\infty}$ and $b>0$ be given. Then the operator $b I+\mathcal{W}[\lambda, \cdot]$ : $\mathcal{C}^{0}([0, T]) \rightarrow \mathcal{C}^{0}([0, T])$ is invertible and its inverse is Lipschitz continuous. In particular the initial value mapping $b I+\mathscr{A}$ (see (3.6)) is increasing and bi-Lipschitz.

As we are dealing with partial differential equations, we should consider both the input and the initial memory configuration $\lambda$ that additionally depend on $x$. If for instance $\lambda(x, \cdot)$ belongs to $\Lambda_{\infty}$ and $u(x, \cdot)$ belongs to $\mathcal{C}^{0}([0, T])$ for (almost) every $x$, then we define

$$
\overline{\mathcal{W}}[\lambda, u](x, t):=\mathcal{W}[\lambda(x, \cdot), u(x, \cdot)](t):=\int_{0}^{\infty} g\left(r, \wp_{r}[\lambda(x, \cdot), u(x, \cdot)](t)\right) d r .
$$

\section{Statement of the main results}

Let $\Omega$ be an open bounded interval of $\mathbb{R}$ and set $\Omega_{T}:=\Omega \times(0, T)$; let us fix an initial memory configuration

$$
\lambda \in L^{2}\left(\Omega ; \Lambda_{\tilde{R}}\right) \quad \text { for some } \tilde{R}>0,
$$

where $\Lambda_{\tilde{R}}$ is introduced in (3.2).

Let $\mathcal{M}\left(\Omega ; \mathcal{C}^{0}([0, T])\right)$ be the Fréchet space of strongly measurable functions $\Omega \rightarrow$ $\mathcal{C}^{0}([0, T])$, i.e. the space of functions $v: \Omega \rightarrow \mathcal{C}^{0}([0, T])$ such that there exists a sequence $v_{n}$ of simple functions with $v_{n} \rightarrow v$ in $\mathcal{C}^{0}([0, T])$ a.e. in $\Omega$.

We fix a constant $b_{\mathcal{F}}>0$ and introduce the operator $\overline{\mathcal{F}}: \mathcal{M}\left(\Omega ; \mathcal{C}^{0}([0, T])\right) \rightarrow$ $\mathcal{M}\left(\Omega ; \mathcal{C}^{0}([0, T])\right)$ in the following way

$$
\overline{\mathcal{F}}(u)(x, t):=\mathcal{F}(u(x, \cdot))(t):=b_{\mathcal{F}} u(x, t)+\mathcal{W}[\lambda(x, \cdot), u(x, \cdot)](t) ;
$$

here $\mathcal{W}$ is the scalar Preisach operator defined in (3.5). 
Now Theorem 3.1 yields that $\mathcal{F}$ is invertible and its inverse is a Lipschitz continuous operator in $\mathcal{C}^{0}([0, T])$. Let us set $\mathcal{G}=\mathcal{F}^{-1}$ and let $L_{\mathcal{G}}$ be the Lipschitz constant of the operator $\mathcal{G}$.

At this point we introduce the operator

$$
\overline{\mathcal{G}}: \mathcal{M}\left(\Omega ; \mathcal{C}^{0}([0, T])\right) \rightarrow \mathcal{M}\left(\Omega ; \mathcal{C}^{0}([0, T])\right) \quad \overline{\mathcal{G}}:=\overline{\mathcal{F}}^{-1}
$$

It turns out that

$$
\overline{\mathcal{G}}(w)(x, t):=\mathcal{G}(w(x, \cdot))(t) \quad \forall w \in \mathcal{M}\left(\Omega ; \mathcal{C}^{0}([0, T])\right) ;
$$

it follows from Theorem 3.1 that $\overline{\mathcal{G}}$ is Lipschitz continuous in the following sense

$$
\begin{aligned}
& \left\|\overline{\mathcal{G}}\left(u_{1}\right)(x, \cdot)-\overline{\mathcal{G}}\left(u_{2}\right)(x, \cdot)\right\|_{\mathcal{C}^{0}([0, T])} \leq L_{\mathcal{G}}\left\|u_{1}(x, \cdot)-u_{2}(x, \cdot)\right\|_{\mathcal{C}^{0}([0, T])} \\
& \text { for any } u_{1}, u_{2} \in \mathcal{M}\left(\Omega ; \mathcal{C}^{0}([0, T])\right) \text {, a.e. in } \Omega
\end{aligned}
$$

The initial conditions for Problem (2.6)

$$
\begin{array}{ll}
E(x, 0):=E_{0}(x) & \text { a.e. in } \mathbb{R}, \\
B(x, 0):=B_{0}(x) & \text { a.e. in } \mathbb{R},
\end{array}
$$

are assumed in the form

$$
\begin{aligned}
& E_{0}(x):=\chi_{\Omega} E_{0}^{1}(x)+\left(1-\chi_{\Omega}\right) E_{0}^{2}(x), \\
& B_{0}(x):=\chi_{\Omega} B_{0}^{1}(x)+\left(1-\chi_{\Omega}\right) B_{0}^{2}(x),
\end{aligned}
$$

as in the following we will assume different regularity for the initial data inside and outside $\Omega$.

The full P.D.E.s system for unknown functions $E, B$ and $H$ reads as follows

$$
\begin{cases}\frac{\partial E}{\partial t}+\chi_{\Omega}\left(E+E_{\mathrm{app}}\right)+\left(1-\chi_{\Omega}\right) J_{\mathrm{ext}}+\frac{\partial H}{\partial x}=0 & \\ \frac{\partial B}{\partial t}+\frac{\partial E}{\partial x}=0 & \text { a.e. in } \mathbb{R} \times(0, T) . \\ H=\chi_{\Omega}\left(\overline{\mathcal{G}}(B)+\gamma \frac{\partial B}{\partial t}\right)+\left(1-\chi_{\Omega}\right) B & \end{cases}
$$

with initial conditions (4.6), where $E_{\text {app }}$ and $J_{\text {ext }}$ are given functions.

For the sake of definiteness, we assume $\Omega=(-1,1)$, fix some $r>1$, and set $K=$ $(-r, r)$.

We first distinguish the case in which the data have compact support; in Section 5 we thus prove the following existence result.

Theorem 4.1. Consider the following assumptions on the initial data

$$
\begin{aligned}
& E_{0}^{1} \in H^{2}(\Omega), \quad B_{0}^{1} \in H^{1}(\Omega) \\
& \operatorname{supp} E_{0}^{2} \subset K \backslash \bar{\Omega}, \quad \operatorname{supp} B_{0}^{2} \subset K \backslash \bar{\Omega}, \quad E_{0}^{2}, B_{0}^{2} \in H^{1}(K \backslash \bar{\Omega})
\end{aligned}
$$


together with the following compatibility conditions

$$
\left\{\begin{array}{l}
\left(\tilde{\mathscr{A}}\left(B_{0}^{1}\right)-\gamma \frac{\partial E_{0}^{1}}{\partial x}\right)\left(-1^{+}\right)=B_{0}^{2}\left(-1^{-}\right), \\
\left(\tilde{\mathscr{A}}\left(B_{0}^{1}\right)-\gamma \frac{\partial E_{0}^{1}}{\partial x}\right)\left(1^{-}\right)=B_{0}^{2}\left(1^{+}\right), \\
E_{0}^{1}\left(-1^{+}\right)=E_{0}^{2}\left(-1^{-}\right), \quad E_{0}^{1}\left(1^{-}\right)=E_{0}^{2}\left(1^{+}\right),
\end{array}\right.
$$

where $\tilde{\mathscr{A}}=(b I+\mathscr{A})^{-1}$ is the initial value mapping associated with $\overline{\mathcal{G}}$, see Theorem 3.1. Moreover assume that

$$
E_{\text {app }} \in H^{1}\left(0, T ; L^{2}(\Omega)\right), \quad J_{\text {ext }} \in H^{1}\left(0, T ; L^{2}(K)\right) .
$$

Then Problem (4.7) has a unique solution

$$
\begin{aligned}
& E \in W^{1, \infty}\left(0, T ; L^{2}(\mathbb{R})\right) \cap L^{2}\left(0, T ; H^{1}(\mathbb{R})\right) \\
& B_{\left.\right|_{\Omega}} \in H^{2}\left(0, T ; L^{2}(\Omega)\right) \\
& B_{\left.\right|_{\mathbb{R} \backslash \bar{\Omega}}} \in W^{1, \infty}\left(0, T ; L^{2}(\mathbb{R} \backslash \bar{\Omega})\right) \\
& H \in H^{1}\left(0, T ; L^{2}(\mathbb{R})\right) \cap L^{\infty}\left(0, T ; H^{1}(\mathbb{R})\right) .
\end{aligned}
$$

Finally in Section 6 we deal with the case of more general data, not necessarily with compact support, and the result we are able to prove is the following.

Theorem 4.2. Consider the following assumptions on the initial data

$$
\begin{array}{ll}
E_{0}^{1} \in H^{2}(\Omega), & B_{0}^{1} \in H^{1}(\Omega) \\
E_{0}^{2} \in H_{\mathrm{loc}}^{1}(\mathbb{R} \backslash \bar{\Omega}), & B_{0}^{2} \in H_{\mathrm{loc}}^{1}(\mathbb{R} \backslash \bar{\Omega}),
\end{array}
$$

together with the compatibility conditions (4.9a), (4.9b) and $(4.9 \mathrm{c})$; moreover assume that

$$
E_{\text {app }} \in H^{1}\left(0, T ; L^{2}(\Omega)\right), \quad J_{\text {ext }} \in H^{1}\left(0, T ; L_{\text {loc }}^{2}(\mathbb{R})\right) .
$$

Then Problem (4.7) has a unique solution such that

$$
\begin{aligned}
& E \in W^{1, \infty}\left(0, T ; L_{\mathrm{loc}}^{2}(\mathbb{R})\right) \cap L^{2}\left(0, T ; H_{\mathrm{loc}}^{1}(\mathbb{R})\right) \\
& B_{\left.\right|_{\Omega}} \in H^{2}\left(0, T ; L^{2}(\Omega)\right) \\
& B_{\left.\right|_{\mathbb{R} \backslash \bar{\Omega}}} \in W^{1, \infty}\left(0, T ; L_{\mathrm{loc}}^{2}(\mathbb{R} \backslash \bar{\Omega})\right) \\
& H \in H^{1}\left(0, T ; L_{\mathrm{loc}}^{2}(\mathbb{R})\right) \cap L^{\infty}\left(0, T ; H_{\mathrm{loc}}^{1}(\mathbb{R})\right) .
\end{aligned}
$$

\section{$5 \quad$ Proof of Theorem 4.1}

The main idea in proving Theorem 4.1 is to use a space discretization scheme together with a fixed point argument. 
Let us fix $R>r+T$; for simplicity (this will be useful in the space discretization procedure) we take $R \in \mathbb{N}$. We prescribe the following "boundary conditions"

$$
H(-R, t)=0, \quad E(R, t)=0 .
$$

- STEP 1: FREEZING. First of all we fix some $Z \in H^{1}\left(0, T ; L^{2}(\Omega)\right)$ with $Z(0, x)=$ $B_{0}^{1}(x)$ a.e. in $\Omega$, and consider $(4.7)$ with $\overline{\mathcal{G}}(B)$ replaced by $\overline{\mathcal{G}}(Z)$, i.e. we look for three functions $E, B, H$ with the regularity outlined in (4.10) and initial conditions (4.6) such that the following holds

$$
\begin{cases}\frac{\partial E}{\partial t}+\chi_{\Omega}\left(E+E_{\mathrm{app}}\right)+\left(1-\chi_{\Omega}\right) J_{\mathrm{ext}}+\frac{\partial H}{\partial x}=0 & \\ \frac{\partial B}{\partial t}+\frac{\partial E}{\partial x}=0 & \text { a.e. in }(-R, R) \times(0, T) . \\ H=\chi_{\Omega}\left(\overline{\mathcal{G}}(Z)+\gamma \frac{\partial B}{\partial t}\right)+\left(1-\chi_{\Omega}\right) B & \end{cases}
$$

- STEP 2: EXISTENCE OF SOlUtions FOR (5.2): SPACE DiSCRETIZATION SCHEME.

We now fix $n \in \mathbb{N}$ and consider the equidistant partition of the interval $[-R, R]$

$$
x_{k}:=\frac{k}{n}, \quad k=-R n,-R n+1, \ldots, R n .
$$

The characteristic function $\chi_{\Omega}$ reduces to

$$
\chi_{k}= \begin{cases}1 & k \in\{-n+1, \ldots, n\} \\ 0 & \text { else }\end{cases}
$$

We introduce the function

$$
F(x, t):=\chi_{\Omega} E_{\text {app }}(x, t)+\left(1-\chi_{\Omega}\right) J_{\text {ext }}(x, t) .
$$

It turns out that

$$
F \in H^{1}\left(0, T ; L^{2}(\mathbb{R})\right) \subset L^{2}\left(\mathbb{R} ; \mathcal{C}^{0}([0, T])\right)
$$

Now we set

$$
\begin{array}{ll}
F_{k}(t)=n \int_{(k-1) / n}^{k / n} F(x, t) d x & k=-R n+1, \ldots, R n, \\
G_{k}(t)=n \int_{(k-1) / n}^{k / n} \overline{\mathcal{G}}(Z)(x, t) d x, & k=-n+2, \ldots, n
\end{array}
$$

and also

$$
\mathcal{D}_{n}:=\{-R n+1, \ldots,-n\} \cup\{n+1, \ldots, R n-1\} .
$$

We now approximate (5.2) by a system of O.D.E.s, where the dot indicates the time derivative. 
Our aim is to find unknown functions $E_{k}, B_{k}, H_{k}$ such that the following holds, for $k=-R n+1, \ldots, R n-1$

$$
\left\{\begin{array}{l}
\dot{E}_{k}+\chi_{k} E_{k}+n\left(H_{k}-H_{k-1}\right)+F_{k}=0 \\
\dot{B}_{k}+n\left(E_{k+1}-E_{k}\right)=0 \\
H_{k}=\chi_{k}\left[G_{k}+\gamma \dot{B}_{k}\right]+\left(1-\chi_{k}\right) B_{k}=0
\end{array}\right.
$$

This is coupled with the boundary conditions

$$
H_{-R n}(t)=0, \quad E_{R n}(t)=0
$$

and initial conditions

$$
\begin{aligned}
& E_{k}(0)=E_{0}^{k}:=E_{0}\left(\frac{k-1}{n}\right) \\
& B_{k}(0)=B_{0}^{k}:=n \int_{(k-1) / n}^{k / n} B_{0}(x) d x .
\end{aligned}
$$

We choose the averages for $B_{k}(0)$ in order to avoid difficulties related to the fact that $B_{0}$ may be discontinuous.

Eliminating $H_{k}, H_{k-1}$ from (5.4a), we rewrite (5.4a)-(5.4b) as a system of $2(2 R n-1)$ equations for $2(2 R n-1)$ unknown functions

$$
\dot{V}=\Phi V+\tilde{F} \quad V=\left(E_{-R n+1}, \ldots, E_{R n-1}, B_{-R n+1}, \ldots, B_{R n-1}\right),
$$

where $\Phi$ is a matrix and $\tilde{F} \in W^{1,2}\left(0, T ; \mathbb{R}^{2(2 R n-1)}\right)$. This is enough to conclude that the system, coupled with (5.5) and (5.6) admits a unique global solution.

In the following, for the sake of simplicity, we denote by $C_{1}, C_{2}, \ldots$ any constant depending possibly on the data but independent on the discretization parameter $n$. We now differentiate (5.4a), (5.4b) and (5.4c) in time, getting

$$
\left\{\begin{array}{l}
\ddot{E}_{k}+\chi_{k} \dot{E}_{k}+n\left(\dot{H}_{k}-\dot{H}_{k-1}\right)+\dot{F}_{k}=0 \\
\ddot{B}_{k}+n\left(\dot{E}_{k+1}-\dot{E}_{k}\right)=0 \\
\dot{H}_{k}=\chi_{k}\left[\dot{G}_{k}+\gamma \ddot{B}_{k}\right]+\left(1-\chi_{k}\right) \dot{B}_{k}=0 .
\end{array}\right.
$$

Now we test (5.7a) by $\dot{E}_{k}$ and (5.7b) by $\dot{H}_{k}$, sum the result and divide by $n$. We have

$$
\begin{aligned}
& \frac{1}{2 n} \frac{d}{d t} \sum_{k=-R n+1}^{R n-1}\left|\dot{E}_{k}\right|^{2}+\frac{1}{n} \sum_{k=-n+1}^{n}\left|\dot{E}_{k}\right|^{2}+\sum_{k=-R n+1}^{R n-1}\left(\dot{H}_{k}-\dot{H}_{k-1}\right) \dot{E}_{k} \\
& +\frac{1}{n} \sum_{k=-R n+1}^{R n-1} \dot{F}_{k} \dot{E}_{k}+\frac{1}{n} \sum_{k=-R n+1}^{R n-1} \ddot{B}_{k} \dot{H}_{k}+\sum_{k=-R n+1}^{R n-1}\left(\dot{E}_{k+1}-\dot{E}_{k}\right) \dot{H}_{k}=0 .
\end{aligned}
$$


We remark that

$$
\begin{aligned}
& \sum_{k=-R n+1}^{R n-1}\left[\left(\dot{H}_{k}-\dot{H}_{k-1}\right) \dot{E}_{k}+\left(\dot{E}_{k+1}-\dot{E}_{k}\right) \dot{H}_{k}\right] \\
= & \sum_{k=-R n+1}^{R n-1}\left[\dot{E}_{k+1} \dot{H}_{k}-\dot{H}_{k-1} \dot{E}_{k}\right]=\left[\dot{E}_{R n} \dot{H}_{R n-1}-\dot{H}_{-R n} \dot{E}_{-R n+1}\right] \stackrel{(5.5)}{=} 0 .
\end{aligned}
$$

Therefore, using $(5.7 \mathrm{c})$, we deduce

$$
\begin{aligned}
& \quad \frac{1}{2 n} \frac{d}{d t} \sum_{k=-R n+1}^{R n-1}\left|\dot{E}_{k}\right|^{2}+\frac{1}{n} \sum_{k=-n+1}^{n}\left|\dot{E}_{k}\right|^{2}+\frac{\gamma}{n} \sum_{k=-n+1}^{n}\left|\ddot{B}_{k}\right|^{2}+\frac{1}{2 n} \frac{d}{d t} \sum_{\mathcal{D}_{n}}\left|\dot{B}_{k}\right|^{2} \\
& \leq \frac{1}{n} \sum_{k=-n+1}^{n} \ddot{B}_{k} \dot{G}_{k}-\frac{1}{n} \sum_{k=-R n+1}^{R n-1} \dot{F}_{k} \dot{E}_{k} \leq \frac{\gamma}{2 n} \sum_{k=-n+1}^{n}\left|\ddot{B}_{k}\right|^{2} \\
& \quad+\frac{1}{2 \gamma n} \sum_{k=-n+1}^{n}\left|\dot{G}_{k}\right|^{2}+\frac{1}{n} \sum_{k=-R n+1}^{R n-1}\left|\dot{F}_{k}\right|^{2}+\frac{1}{n} \sum_{k=-R n+1}^{R n-1}\left|\dot{E}_{k}\right|^{2} .
\end{aligned}
$$

Now, using (5.3) we deduce in particular that

$$
\begin{aligned}
& \frac{1}{2 n} \frac{d}{d t} \sum_{k=-R n+1}^{R n-1}\left|\dot{E}_{k}\right|^{2}+\frac{1}{2 n} \frac{d}{d t} \sum_{\mathcal{D}_{n}}\left|\dot{B}_{k}\right|^{2} \\
\leq & \int_{-R}^{R} F^{2}(x, t) d x+\frac{1}{n} \sum_{k=-R n+1}^{R n-1}\left|\dot{E}_{k}\right|^{2}+\frac{1}{n} \sum_{\mathcal{D}_{n}}\left|\dot{B}_{k}\right|^{2} .
\end{aligned}
$$

The Gronwall lemma then yields

$$
\frac{1}{n}\left(\sum_{k=-R n+1}^{R n-1}\left|\dot{E}_{k}\right|^{2}+\sum_{\mathcal{D}_{n}}\left|\dot{B}_{k}\right|^{2}\right) \leq \frac{C_{1}}{n}\left[\sum_{k=-R n+1}^{R n-1}\left|\dot{E}_{k}(0)\right|^{2}+\sum_{\mathcal{D}_{n}}\left|\dot{B}_{k}(0)\right|^{2}\right]+C_{2} .
$$

We have now to show that, due to our assumptions on the data, the term

$$
\frac{1}{n} \sum_{k=-R n+1}^{R n-1}\left|\dot{E}_{k}(0)\right|^{2}+\frac{1}{n} \sum_{\mathcal{D}_{n}}\left|\dot{B}_{k}(0)\right|^{2}
$$

can be controlled by a constant independent of the discretization parameter $n$. First of all, by comparison and using (5.4a) we have that

$$
\begin{aligned}
\frac{1}{n} \sum_{k=-R n+1}^{R n-1}\left|\dot{E}_{k}(0)\right|^{2} \leq \frac{1}{n} \sum_{k=-n+1}^{n}\left|E_{k}(0)\right|^{2} & +n \sum_{k=-R n+1}^{R n-1}\left|H_{k}(0)-H_{k-1}(0)\right|^{2} \\
& +\frac{1}{n} \sum_{k=-R n+1}^{R n-1}\left|F_{k}(0)\right|^{2} .
\end{aligned}
$$


As $E_{0}^{1} \in H^{2}(\Omega)$, the first term on the right-hand side of the previous inequality can be controlled while the third one is controlled due to (5.3).

To estimate the second term, we first notice that for $k \in \mathcal{D}_{n}$, we have

$$
H_{k}(0)=B_{k}(0),
$$

while for $k^{\prime} \in\{-n+1, \ldots, n\}$, equation (5.4c) yields

$$
H_{k^{\prime}}(0)=G_{k^{\prime}}(0)+\gamma \dot{B}_{k^{\prime}}(0)=G_{k^{\prime}}(0)-\gamma n\left(E_{k^{\prime}+1}(0)-E_{k^{\prime}}(0)\right) .
$$

We have by (3.3) for $x \in \Omega$ that

$$
\overline{\mathcal{G}}(Z)(x, 0)=\tilde{\mathscr{A}}\left(B_{0}^{1}(x)\right)
$$

hence

$$
G_{k^{\prime}}(0)=n \int_{\left(k^{\prime}-1\right) / n}^{k^{\prime} / n} \tilde{\mathscr{A}}\left(B_{0}^{1}(x)\right) d x \quad k^{\prime} \in\{-n+2, \ldots, n\} .
$$

This yields

$$
\begin{aligned}
& n \sum_{k=-R n+1}^{R n-1}\left|H_{k}(0)-H_{k-1}(0)\right|^{2}=n \sum_{k=-R n+1}^{-n}\left|B_{k}(0)-B_{k-1}(0)\right|^{2}+n\left|H_{-n+1}(0)-H_{-n}(0)\right|^{2} \\
& +n \sum_{k=-n+2}^{n}\left|G_{k}(0)-G_{k-1}(0)-\gamma n\left(E_{k+1}(0)-2 E_{k}(0)+E_{k-1}(0)\right)\right|^{2} \\
& +n\left|H_{n+1}(0)-H_{n}(0)\right|^{2}+n \sum_{k=n+2}^{R n-1}\left|B_{k}(0)-B_{k-1}(0)\right|^{2} .
\end{aligned}
$$

Two terms deserve special attention. First

$$
\begin{aligned}
& H_{-n+1}(0)-H_{-n}(0)=G_{-n+1}(0)-\gamma n\left(E_{-n+2}(0)-E_{-n+1}(0)\right)-B_{-n}(0) \\
= & n \int_{-1}^{-1+\frac{1}{n}} \tilde{\mathscr{A}}\left(B_{0}^{1}(x)\right) d x-\gamma n\left(E_{0}^{1}\left(-1+\frac{1}{n}\right)-E_{0}^{1}(-1)\right)-n \int_{-1-\frac{1}{n}}^{-1} B_{0}^{2}(x) d x \\
= & n \int_{-1}^{-1+\frac{1}{n}} \tilde{\mathscr{A}}\left(B_{0}^{1}(x)\right) d x-\gamma n \int_{-1}^{-1+\frac{1}{n}} \frac{\partial E_{0}^{1}}{\partial x}(x) d x-n \int_{-1-\frac{1}{n}}^{-1} B_{0}^{2}(x) d x \\
= & n \int_{-1}^{-1+\frac{1}{n}}\left[\tilde{\mathscr{A}}\left(B_{0}^{1}(x)\right)-\tilde{\mathscr{A}}\left(B_{0}^{1}\left(-1^{+}\right)\right)\right] d x-\gamma n \int_{-1}^{-1+\frac{1}{n}}\left[\frac{\partial E_{0}^{1}}{\partial x}(x)-\frac{\partial E_{0}^{1}}{\partial x}\left(-1^{+}\right)\right] d x \\
& -n \int_{-1-\frac{1}{n}}^{-1}\left[B_{0}^{2}(x)-B_{0}^{2}\left(-1^{-}\right)\right] d x+\tilde{\mathscr{A}}\left(B_{0}^{1}\left(-1^{+}\right)\right)-\gamma \frac{\partial E_{0}^{1}}{\partial x}\left(-1^{+}\right)-B_{0}^{2}\left(-1^{-}\right) .
\end{aligned}
$$

Using the compatibility condition (4.9a), we obtain

$$
\begin{aligned}
& \left|H_{-n+1}(0)-H_{-n}(0)\right| \leq n \int_{-1}^{-1+\frac{1}{n}}\left|\tilde{\mathscr{A}}\left(B_{0}^{1}(x)\right)-\tilde{\mathscr{A}}\left(B_{0}^{1}\left(-1^{+}\right)\right)\right| d x \\
& +\gamma n \int_{-1}^{-1+\frac{1}{n}}\left|\frac{\partial E_{0}^{1}}{\partial x}(x)-\frac{\partial E_{0}^{1}}{\partial x}\left(-1^{+}\right)\right| d x+n \int_{-1-\frac{1}{n}}^{-1}\left|B_{0}^{2}(x)-B_{0}^{2}\left(-1^{-}\right)\right| d x .
\end{aligned}
$$


The initial value mapping $\tilde{\mathscr{A}}$ is Lipschitz continuous. Hence,

$$
\begin{aligned}
& n \int_{-1}^{-1+\frac{1}{n}}\left|\tilde{\mathscr{A}}\left(B_{0}^{1}(x)\right)-\tilde{\mathscr{A}}\left(B_{0}^{1}\left(-1^{+}\right)\right)\right| d x \leq C_{3} n \int_{-1}^{-1+\frac{1}{n}} \int_{-1}^{x}\left|\frac{\partial B_{0}^{1}}{\partial \xi}(\xi)\right| d \xi d x \\
= & C_{3} n \int_{-1}^{-1+\frac{1}{n}}\left|\frac{\partial B_{0}^{1}}{\partial \xi}(\xi)\right|\left(\int_{\xi}^{-1+\frac{1}{n}} d x\right) d \xi \\
\leq & C_{3} \int_{-1}^{-1+\frac{1}{n}}\left|\frac{\partial B_{0}^{1}}{\partial \xi}(\xi)\right| d \xi \leq \frac{C_{3}}{\sqrt{n}}\left(\int_{-1}^{-1+\frac{1}{n}}\left|\frac{\partial B_{0}^{1}}{\partial x}\right|^{2} d x\right)^{1 / 2} .
\end{aligned}
$$

The other terms are treated similarly, so that

$$
\left|H_{-n+1}(0)-H_{-n}(0)\right| \leq \frac{C_{4}}{\sqrt{n}}\left[\int_{-1}^{-1+\frac{1}{n}}\left(\left|\frac{\partial B_{0}^{1}}{\partial x}\right|^{2}+\left|\frac{\partial^{2} E_{0}^{1}}{\partial x^{2}}\right|^{2}\right) d x+\int_{-1-\frac{1}{n}}^{-1}\left|\frac{\partial B_{0}^{2}}{\partial x}\right|^{2} d x\right] .
$$

We can estimate in a similar way the term $n\left|H_{n+1}(0)-H_{n}(0)\right|^{2}$, using this time the compatibility condition (4.9b).

The remaining terms can be estimated in a more standard way using the regularity of the data (4.8), and we obtain

$$
\frac{1}{n} \sum_{k=-R n+1}^{R n-1}\left|\dot{E}_{k}(0)\right|^{2} \leq C_{5}
$$

On the other hand, by comparison, using (5.4b)

$$
\frac{1}{n} \sum_{\mathcal{D}_{n}}\left|\dot{B}_{k}(0)\right|^{2} \leq n \sum_{\mathcal{D}_{n}}\left|E_{k+1}(0)-E_{k}(0)\right|^{2}
$$

and this can be controlled using the fact that $E_{0}^{2} \in H^{1}(K)$. Therefore, summing up we deduce

$$
\begin{aligned}
& \max _{0 \leq t \leq T}\left[\frac{1}{n} \sum_{k=-R n+1}^{R n-1}\left|\dot{E}_{k}(t)\right|^{2}+\frac{1}{n} \sum_{\mathcal{D}_{n}}\left|\dot{B}_{k}(t)\right|^{2}\right] \\
& +\int_{0}^{T}\left(\frac{1}{n} \sum_{k=-n+1}^{n}\left|\dot{E}_{k}(t)\right|^{2}\right) d t+\frac{\gamma}{n} \int_{0}^{T}\left(\sum_{k=-n+1}^{n}\left|\ddot{B}_{k}(t)\right|^{2}\right) d t \leq C_{6} .
\end{aligned}
$$

We have as a consequence that

$$
\max _{0 \leq t \leq T} \frac{1}{n} \sum_{k=-R n+1}^{R n-1}\left|\dot{B}_{k}(t)\right|^{2} \leq C_{7}
$$

and by comparison,

$$
\max _{0 \leq t \leq T} n \sum_{k=-R n+1}^{R n-1}\left|E_{k+1}(t)-E_{k}(t)\right|^{2} \leq C_{8}
$$


At this point, with the intention to let $n$ tend to $\infty$, we define the following interpolates

$$
\begin{aligned}
& E^{(n)}(x, t)=E_{k}(t)+n\left(x-\frac{(k-1)}{n}\right)\left[E_{k+1}(t)-E_{k}(t)\right] \\
& H^{(n)}(x, t)=H_{k-1}(t)+n\left(x-\frac{(k-1)}{n}\right)\left[H_{k}(t)-H_{k-1}(t)\right] \\
& \bar{E}^{(n)}(x, t)=E_{k}(t) \\
& \bar{H}^{(n)}(x, t)=H_{k}(t) \\
& \bar{B}^{(n)}(x, t)=B_{k}(t) \\
& \bar{G}^{(n)}(x, t)=G_{k}(t) \\
& \bar{\chi}^{(n)}(x)=\chi_{k} \\
& \bar{F}^{(n)}(x, t)=F_{k}(t)
\end{aligned}
$$

for $x \in\left(\frac{k-1}{n}, \frac{k}{n}\right], k=-R n+1, \ldots, R n$ and $t \in[0, T]$. Therefore Problem (5.4a)-(5.4c) can be rewritten as

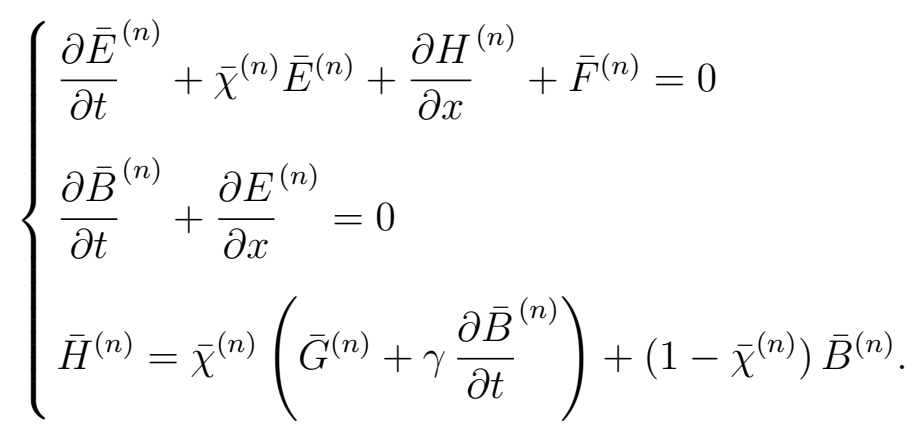

The a priori estimate (5.8) gives

$$
\begin{aligned}
& \max _{0 \leq t \leq T}\left(\left\|\frac{\partial E^{(n)}}{\partial t}(t)\right\|_{L^{2}(-R, R)}^{2}+\left\|{\frac{\partial \bar{E}^{(n)}}{\partial t}}^{(t)}\right\|_{L^{2}(-R, R)}^{2}+\left\|{\frac{\partial \bar{B}^{(n)}}{\partial t}}^{(t)}\right\|_{L^{2}((-R, R) \backslash \bar{\Omega})}^{2}\right)
\end{aligned}
$$

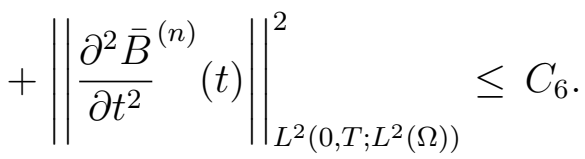

At this point, by comparison, (5.3), (5.10a) and (5.11) give

$$
\max _{0 \leq t \leq T}\left\|\frac{\partial H^{(n)}}{\partial x}(t)\right\|_{L^{2}(-R, R)}^{2} \leq C_{9} .
$$

On the other hand (5.11) entails

$$
\left\|{\frac{\partial \bar{B}^{(n)}}{\partial t}}^{(t)}\right\|_{L^{2}\left(0, T ; L^{2}(-R, R)\right)}^{2} \leq C_{10},
$$


and, by comparison, using (5.10b) we deduce

$$
\left\|{\frac{\partial E^{(n)}}{\partial x}}^{(t)}\right\|_{L^{2}\left(0, T ; L^{2}(-R, R)\right)}^{2} \leq C_{11} .
$$

Combining the above estimates and possible selecting a suitable subsequence of $n \rightarrow$ $\infty$, we find that there exist functions $E, B, H$ in the appropriate Sobolev spaces such that the following convergences take place:

$$
\begin{array}{rlr}
\left.\frac{\partial E^{(n)}}{\partial t} \rightarrow \frac{\partial E}{\partial t}, \quad \frac{\partial H^{(n)}}{\partial x} \rightarrow \frac{\partial H}{\partial x}\right\} & \text { weakly-star in } L^{\infty}\left(0, T ; L^{2}(-R, R)\right), \\
\left.\frac{\partial^{2} \bar{B}^{(n)}}{\partial t^{2}} \rightarrow \frac{\partial^{2} B}{\partial t^{2}}\right\} & \text { weakly in } L^{2}\left(0, T ; L^{2}(\Omega)\right), \\
\left.\frac{\partial \bar{B}^{(n)}}{\partial t} \rightarrow \frac{\partial B}{\partial t}, \quad \frac{\partial E}{\partial x}^{(n)} \rightarrow \frac{\partial E}{\partial x}\right\} & \text { weakly in } L^{2}\left(0, T ; L^{2}(-R, R)\right) \\
E^{(n)} \rightarrow E & \text { uniformly in } \mathcal{C}([-R, R] \times[0, T]) .
\end{array}
$$

Note that

$$
\left|E^{(n)}(x, t)-\bar{E}^{(n)}(x, t)\right|^{2} \leq \sum_{k=-R n+1}^{R n-1}\left|E_{k+1}(t)-E_{k}(t)\right|^{2} \stackrel{(5.9)}{\leq} \frac{C_{8}}{n},
$$

hence

$$
\frac{\partial \bar{E}^{(n)}}{\partial t} \rightarrow \frac{\partial E}{\partial t} \quad \text { weakly-star in } L^{\infty}\left(0, T ; L^{2}(-R, R)\right) .
$$

Therefore we can pass to the limit in (5.10a)-(5.10c) to see that (5.2) is satisfied a.e. in $(-R, R) \times(0, T)$ and the solution has the regularity outlined in (4.10).

- STEP 3: FINITE SPEED OF PROPAGATION.

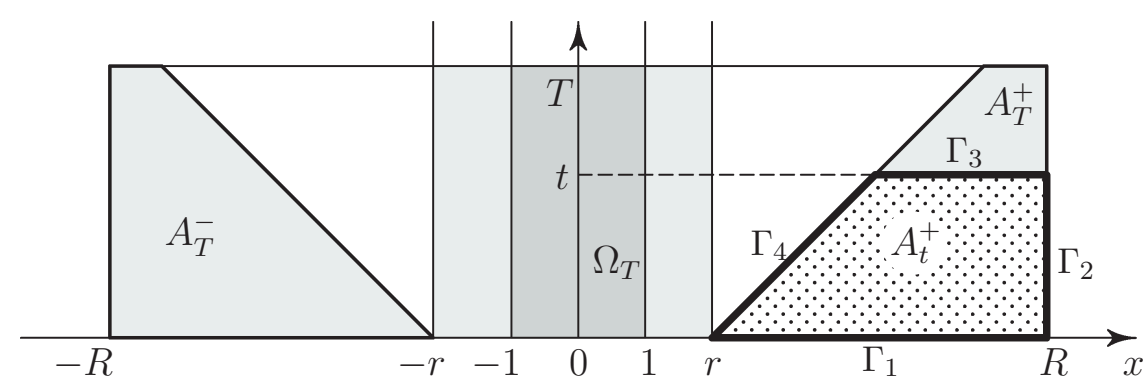

Figure 1: Finite speed of propagation outside $\Omega$.

In order to find solutions to (5.2), we used a space discretization scheme; by doing that, we actually solved a boundary value problem in the domain $(-R, R) \times(0, T)$. To complete our existence result we have actually to show that the solution we found solves also our original model problem. 
Our argument is illustrated on Figure 1. We define the sets

$$
\begin{aligned}
& A_{T}^{+}=\{(x, t) \in \mathbb{R} \times(0, T) ; t \in(0, T), r+t<x<R\} \\
& A_{T}^{-}=\{(x, t) \in \mathbb{R} \times(0, T) ; t \in(0, T),-R<x<-r-t\}
\end{aligned}
$$

with the intention to prove that $E \equiv H \equiv 0$ in $A_{T}^{+} \cup A_{T}^{-}$. In $A_{T}^{+}, E$ and $H$ are solutions of the linear wave equation

$$
\left\{\begin{array}{l}
\frac{\partial E}{\partial t}+\frac{\partial H}{\partial x}=0 \\
\frac{\partial H}{\partial t}+\frac{\partial E}{\partial x}=0
\end{array}\right.
$$

hence satisfy the energy balance

$$
\frac{\partial}{\partial t}\left(\frac{1}{2}\left(E^{2}+H^{2}\right)\right)+\frac{\partial}{\partial x}(E H)=0 .
$$

We now proceed as in [4] and integrate (5.13) over the set $A_{t}^{+}=A_{T}^{+} \cap(\mathbb{R} \times(0, t))$ with some fixed $t \in(0, T)$. Using Green's formula, we obtain

$$
\int_{A_{t}^{+}} \frac{\partial}{\partial t}\left(\frac{1}{2}\left(E^{2}+H^{2}\right)\right)+\frac{\partial}{\partial x}(E H) d x d t=\int_{\partial A_{t}^{+}}\left(E H n_{1}+\frac{1}{2}\left(E^{2}+H^{2}\right) n_{2}\right) d s
$$

where $\vec{n}=\left(n_{1}, n_{2}\right)$ is the unit outward normal vector to $\partial A_{t}^{+}$. The boundary of $A_{t}^{+}$ consists of four parts: $\partial A_{t}^{+}=\Gamma_{1} \cup \Gamma_{2} \cup \Gamma_{3} \cup \Gamma_{4}$, with $\vec{n}=(0,-1)$ on $\Gamma_{1}, \vec{n}=(1,0)$ on $\Gamma_{2}, \vec{n}=(0,1)$ on $\Gamma_{3}$, and $\vec{n}=\left(-\frac{1}{\sqrt{2}}, \frac{1}{\sqrt{2}}\right)$ on $\Gamma_{4}$. We have

$$
\begin{aligned}
& \int_{\Gamma_{1}}\left(E H n_{1}+\frac{1}{2}\left(E^{2}+H^{2}\right) n_{2}\right) d s=-\int_{r}^{R} \frac{1}{2}\left[\left(E_{0}^{2}\right)^{2}(x)+\left(B_{0}^{2}\right)^{2}(x)\right] d x=0 \\
& \int_{\Gamma_{2}}\left(E H n_{1}+\frac{1}{2}\left(E^{2}+H^{2}\right) n_{2}\right) d s=\int_{0}^{t} E(R, \tau) H(R, \tau) d \tau=0 \\
& \int_{\Gamma_{3}}\left(E H n_{1}+\frac{1}{2}\left(E^{2}+H^{2}\right) n_{2}\right) d s=\int_{r+t}^{R} \frac{1}{2}\left(E^{2}(x, t)+H^{2}(x, t)\right) d x \geq 0 \\
& \int_{\Gamma_{4}}\left(E H n_{1}+\frac{1}{2}\left(E^{2}+H^{2}\right) n_{2}\right) d s=\frac{1}{2 \sqrt{2}} \int_{0}^{t}(E-H)^{2}(r+\tau, \tau) d \tau \geq 0 .
\end{aligned}
$$

Comparing these identities with (5.13) and (5.14), we see that $E=H=0$ on $\Gamma_{3}$. As $t \in(0, T)$ has been arbitrary, we obtain the desired result $E=H=0$ in $A_{T}^{+}$.

The argument in $A_{T}^{-}$is fully analogous.

- STEP 4: FIXED POINT.

Let us come back to our original problem. For any $Z \in H^{1}\left(0, T ; L^{2}(\Omega)\right)$ we found a unique solution $(E, B, H)$ to (5.2) with the regularity outlined in (4.10). Thus we can introduce the following closed subspace $S$ of $H^{1}\left(0, T ; L^{2}(\Omega)\right)$

$$
S:=\left\{Z \in H^{1}\left(0, T ; L^{2}(\Omega)\right): Z(0, x)=B_{0}^{1}(x)\right\},
$$


and an operator $J: S \rightarrow S$ which associates with every fixed datum $Z \in S$ the quantity $B \in S$ of the corresponding solution $(E, B, H)$. At this point we take two different data $Z_{1}, Z_{2} \in S$ and consider the corresponding solutions to $(5.2),\left(E_{1}, B_{1}, H_{1}\right)$ and $\left(E_{2}, B_{2}, H_{2}\right)$, respectively associated with $Z_{1}$ and $Z_{2}$. We have $B_{1}:=J\left(Z_{1}\right)$ and $B_{2}:=J\left(Z_{2}\right)$ and moreover we obtain

$$
\left\{\begin{array}{l}
\frac{\partial}{\partial t}\left(E_{1}-E_{2}\right)+\chi_{\Omega}\left(E_{1}-E_{2}\right)+\frac{\partial}{\partial x}\left(H_{1}-H_{2}\right)=0 \\
\frac{\partial}{\partial t}\left(B_{1}-B_{2}\right)+\frac{\partial}{\partial x}\left(E_{1}-E_{2}\right)=0 \\
H_{1}-H_{2}=\chi_{\Omega}\left[\overline{\mathcal{G}}\left(Z_{1}\right)-\overline{\mathcal{G}}\left(Z_{2}\right)+\gamma \frac{\partial}{\partial t}\left(B_{1}-B_{2}\right)\right]+\left(1-\chi_{\Omega}\right)\left(B_{1}-B_{2}\right) .
\end{array}\right.
$$

We test $(5.15 \mathrm{a})$ by $\left(E_{1}-E_{2}\right)$ and $(5.15 \mathrm{~b})$ by $\left(H_{1}-H_{2}\right)$ and then sum the result; taking into account that the terms

$$
\int_{\mathbb{R}} \frac{\partial}{\partial x}\left(H_{1}-H_{2}\right)\left(E_{1}-E_{2}\right) d x \quad \int_{\mathbb{R}} \frac{\partial}{\partial x}\left(E_{1}-E_{2}\right)\left(H_{1}-H_{2}\right) d x
$$

cancel out, we deduce, using (5.15c)

$$
\begin{aligned}
& \frac{1}{2} \frac{d}{d t} \int_{\mathbb{R}}\left|E_{1}-E_{2}\right|^{2} d x+\int_{\Omega}\left|E_{1}-E_{2}\right|^{2} d x+\frac{3}{4} \gamma \int_{\Omega}\left|\frac{\partial}{\partial t}\left(B_{1}-B_{2}\right)\right|^{2} d x \\
& +\frac{1}{2} \frac{d}{d t} \int_{\mathbb{R} \backslash \Omega}\left|B_{1}-B_{2}\right|^{2} d x \leq \frac{1}{\gamma} \int_{\Omega}\left|\overline{\mathcal{G}}\left(Z_{1}\right)-\overline{\mathcal{G}}\left(Z_{2}\right)\right|^{2} d x
\end{aligned}
$$

We remark that this last estimate together with the causality of the operator $\overline{\mathcal{G}}$ entails that (5.2) admits a unique solution, for any fixed $Z \in H^{1}\left(0, T ; L^{2}(\Omega)\right)$.

We now set

$$
D(t):=\frac{1}{2}\left[\int_{\mathbb{R}}\left|E_{1}-E_{2}\right|^{2} d x+\int_{\mathbb{R} \backslash \Omega}\left|B_{1}-B_{2}\right|^{2} d x\right]
$$

and we remark that $D(0)=0$. Thus in particular we deduce

$$
\frac{d D}{d t}+\frac{3}{4} \gamma \int_{\Omega}\left|\frac{\partial}{\partial t}\left(B_{1}-B_{2}\right)\right|^{2} d x \leq \frac{1}{\gamma} \int_{\Omega}\left|\overline{\mathcal{G}}\left(Z_{1}\right)-\overline{\mathcal{G}}\left(Z_{2}\right)\right|^{2} d x
$$

On the other hand

$$
\begin{aligned}
\int_{\Omega}\left[\overline{\mathcal{G}}\left(Z_{1}\right)(x, t)-\overline{\mathcal{G}}\left(Z_{2}\right)(x, t)\right]^{2} d x & \stackrel{(4.5)}{\leq} L_{\mathcal{G}}^{2} \int_{\Omega}\left\|Z_{1}(x, \cdot)-Z_{2}(x, \cdot)\right\|_{\mathcal{C}^{0}([0, t])}^{2} d x \\
& \leq L_{\mathcal{G}}^{2} \int_{\Omega}\left(\int_{0}^{t}\left|\frac{\partial}{\partial \tau}\left(Z_{1}-Z_{2}\right)\right|(x, \tau) d \tau\right)^{2} d x \\
& \leq L_{\mathcal{G}}^{2} t \int_{0}^{t} \int_{\Omega}\left|\frac{\partial}{\partial \tau}\left(Z_{1}-Z_{2}\right)\right|^{2}(x, \tau) d x d \tau
\end{aligned}
$$


where we used the fact that $Z_{1}(0, x)=Z_{2}(0, x)$, a.e. in $\Omega$, as $Z_{1}, Z_{2} \in S$.

We now introduce an equivalent norm on $H^{1}\left(0, T ; L^{2}(\Omega)\right)$ : for all $z \in H^{1}\left(0, T ; L^{2}(\Omega)\right)$

$$
\|z\|:=\left(\|z(0)\|_{L^{2}(\Omega)}^{2}+\int_{0}^{T} \exp \left(-\frac{2 L_{\mathcal{G}}^{2} t^{2}}{\gamma^{2}}\right)\left\|\frac{\partial z}{\partial t}\right\|_{L^{2}(\Omega)}^{2}(t) d t\right)^{1 / 2} .
$$

At this point we divide (5.16) by $\gamma$, multiply the result by $\exp \left(-\frac{2 L_{\mathcal{G}}^{2} t^{2}}{\gamma^{2}}\right)$ and integrate in time, for $t \in(0, T)$. We first remark that

$$
\begin{aligned}
& \int_{0}^{T} \exp \left(-\frac{2 L_{\mathcal{G}}^{2} t^{2}}{\gamma^{2}}\right) \frac{d D}{d t}(t) d t=\exp \left(-\frac{2 L_{\mathcal{G}}^{2} T^{2}}{\gamma^{2}}\right) D(T) \\
& +\int_{0}^{T} D(t) \exp \left(-\frac{2 L_{\mathcal{G}}^{2} t^{2}}{\gamma^{2}}\right) \frac{4 L_{\mathcal{G}}^{2} t}{\gamma^{2}} d t \geq 0
\end{aligned}
$$

and therefore we have

$$
\begin{aligned}
\frac{3}{4}\left\|\left|J\left(Z_{1}\right)-J\left(Z_{2}\right) \|\right|^{2} \leq\right. & \int_{0}^{T} \exp \left(-\frac{2 L_{\mathcal{G}}^{2} t^{2}}{\gamma^{2}}\right) \frac{L_{\mathcal{G}}^{2} t}{\gamma^{2}} \int_{0}^{t} \int_{\Omega}\left|\frac{\partial}{\partial \tau}\left(Z_{1}-Z_{2}\right)\right|^{2}(x, \tau) d x d \tau \\
= & -\frac{1}{4} \exp \left(-\frac{2 L_{\mathcal{G}}^{2} T^{2}}{\gamma^{2}}\right) \int_{0}^{T} \int_{\Omega}\left|\frac{\partial}{\partial t}\left(Z_{1}-Z_{2}\right)\right|^{2}(x, t) d x d t \\
& +\frac{1}{4} \int_{0}^{T} \exp \left(-\frac{2 L_{\mathcal{G}}^{2} t^{2}}{\gamma^{2}}\right) \int_{\Omega}\left|\frac{\partial}{\partial t}\left(Z_{1}-Z_{2}\right)\right|^{2}(x, t) d x d t
\end{aligned}
$$

which in turn gives

$$
\left\|\mid J\left(Z_{1}\right)-J\left(Z_{2}\right)\right\|^{2} \leq \frac{1}{3}\left\|Z_{1}-Z_{2}\right\|^{2} .
$$

Hence $J$ is a contraction on the closed subset $S$ of $H^{1}\left(0, T ; L^{2}(\Omega)\right)$, which finishes the proof.

\section{$6 \quad$ Proof of Theorem 4.2}

We now deal with the general case.

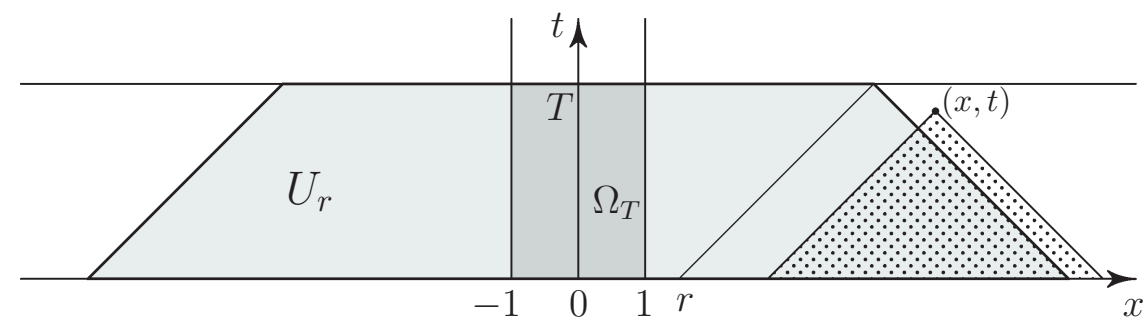

Figure 2: Set $U_{r}$ and domain of dependence for $(x, t)$ outside $U_{r}$. 
For data as in Theorem 4.2, we define sequences indexed by $n \in \mathbb{N}$ of truncated data with supports in $(-2 n, 2 n)$ for $n \rightarrow \infty$ as

$$
\begin{gathered}
J_{\text {ext }}^{n}(x, t)=\chi_{(-n, n)}(x) J_{\text {ext }}(x, t), \\
\left(E_{0}^{2}\right)^{n}(x)= \begin{cases}E_{0}^{2}(x) & \text { for } x \in(-n, n) \\
\left(2-\frac{x}{n}\right) E_{0}^{2}(n) & \text { for } x \in[n, 2 n) \\
\left(2+\frac{x}{n}\right) E_{0}^{2}(-n) & \text { for } x \in(-2 n,-n] \\
0 & \text { for }|x| \geq 2 n,\end{cases} \\
\left(B_{0}^{2}\right)^{n}(x)= \begin{cases}B_{0}^{2}(x) & \text { for } x \in(-n, n) \\
\left(2-\frac{x}{n}\right) B_{0}^{2}(n) & \text { for } x \in[n, 2 n) \\
\left(2+\frac{x}{n}\right) B_{0}^{2}(-n) & \text { for } x \in(-2 n,-n] \\
0 & \text { for }|x| \geq 2 n,\end{cases}
\end{gathered}
$$

By Theorem 4.1, there exists a sequence of solutions $\left\{\left(E^{n}, B^{n}, H^{n}\right)\right\}_{n \in \mathbb{N}}$ associated with these data. We now refer again to the Courant-Hilbert trick and show that on every domain

$$
U_{r}=\{(x, t) \in \mathbb{R} \times(0, T) ; 0 \leq t \leq T,-r-2 T+t \leq x \leq r+2 T-t\}
$$

(see Fig. 2) with an arbitrarily fixed parameter $r>1$, all these solutions with $n>$ $r+2 T$ coincide.

Let us take two solutions $\left(E^{j}, B^{j}, H^{j}\right)$ and $\left(E^{k}, B^{k}, H^{k}\right)$, with $j \neq k, j, k>r+2 T$. We set $\bar{E}:=E^{j}-E^{k}, \bar{B}:=B^{j}-B^{k}, \bar{H}:=H^{j}-H^{k}, \bar{G}:=\overline{\mathcal{G}}\left(B^{j}\right)-\overline{\mathcal{G}}\left(B^{k}\right)$. Then we obtain

$$
\left\{\begin{array}{l}
\frac{\partial \bar{E}}{\partial t}+\chi_{\Omega} \bar{E}+\frac{\partial \bar{H}}{\partial x}=0 \\
\frac{\partial \bar{B}}{\partial t}+\frac{\partial \bar{E}}{\partial x}=0 \\
\bar{H}=\chi_{\Omega}\left(\bar{G}+\gamma \frac{\partial \bar{B}}{\partial t}\right)+\left(1-\chi_{\Omega}\right) \bar{B}
\end{array}\right.
$$

We test (6.1a) by $\bar{E},(6.1 \mathrm{~b})$ by $\bar{H}$ and integrate over $U_{r}$. We obtain

$$
\begin{aligned}
& \int_{\Omega_{T}}\left(\bar{E}^{2}+\gamma\left(\frac{\partial \bar{B}}{\partial t}\right)^{2}+\bar{G} \frac{\partial \bar{B}}{\partial t}\right) d x d t \\
& +\int_{U_{r}}\left[\frac{\partial}{\partial t}\left(\frac{1}{2}\left(\bar{E}^{2}+\left(1-\chi_{\Omega}\right) \bar{B}^{2}\right)\right)+\frac{\partial}{\partial x}(\bar{E} \bar{H})\right] d x d t=0 .
\end{aligned}
$$


The set $\partial U_{r}$ consists of four straight segments, $\tilde{\Gamma}_{1}, \tilde{\Gamma}_{2}, \tilde{\Gamma}_{3}, \tilde{\Gamma}_{4}$, with endpoints respectively $(-r-2 T, 0)$ and $(r+2 T, 0),(r+2 T, 0)$ and $(r+T, T),(r+T, T)$ and $(-r-T, T)$, $(-r-T, T)$ and $(-r-2 T, 0)$, see Fig. 2.

Now, the Green formula yields

$$
\begin{aligned}
& \int_{U_{r}}\left[\frac{\partial}{\partial t}\left(\frac{1}{2}\left(\bar{E}^{2}+\left(1-\chi_{\Omega}\right) \bar{B}^{2}\right)\right)+\frac{\partial}{\partial x}(\bar{E} \bar{H})\right] d x d t=-\int_{\tilde{\Gamma}_{1}} \frac{1}{2}\left(\bar{E}^{2}+\left(1-\chi_{\Omega}\right) \bar{B}^{2}\right) d s \\
& +\int_{\tilde{\Gamma}_{2}}\left[\frac{1}{\sqrt{2}}(\bar{E} \bar{H})+\frac{1}{2 \sqrt{2}}\left(\bar{E}^{2}+\left(1-\chi_{\Omega}\right) \bar{B}^{2}\right)\right] d s+\int_{\tilde{\Gamma}_{3}} \frac{1}{2}\left(\bar{E}^{2}+\left(1-\chi_{\Omega}\right) \bar{B}^{2}\right) d s \\
& +\int_{\tilde{\Gamma}_{4}}\left[-\frac{1}{\sqrt{2}}(\bar{E} \bar{H})+\frac{1}{2 \sqrt{2}}\left(\bar{E}^{2}+\left(1-\chi_{\Omega}\right) \bar{B}^{2}\right)\right] d s .
\end{aligned}
$$

Now the integral over $\tilde{\Gamma}_{1}$ vanishes as the initial data for $\left(E^{j}, B^{j}, H^{j}\right)$ and $\left(E^{k}, B^{k}, H^{k}\right)$ coincide while the integral over $\tilde{\Gamma}_{3}$ yields a nonnegative contribution. Finally, taking into account that

$$
\tilde{\Gamma}_{2} \cap \Omega_{T}=\emptyset \quad \tilde{\Gamma}_{4} \cap \Omega_{T}=\emptyset,
$$

we have that the integrals over $\tilde{\Gamma}_{2}$ and $\tilde{\Gamma}_{4}$ give a nonnegative contribution, as $\bar{H}=\bar{B}$. At this point (6.2) entails in particular

$$
\int_{\Omega_{T}}\left(\gamma\left(\frac{\partial \bar{B}}{\partial t}\right)^{2}+\bar{G} \frac{\partial \bar{B}}{\partial t}\right) d x d t \leq 0
$$

This in turns gives, working as in (5.17)

$$
\frac{\gamma}{2} \int_{\Omega_{T}}\left|\frac{\partial \bar{B}}{\partial t}\right|^{2} d x d t \leq \frac{1}{2 \gamma} \int_{0}^{T} \int_{\Omega}|\bar{G}|^{2} d x d t \leq \int_{0}^{T} \frac{L_{\mathcal{G}}^{2}}{2 \gamma} t \int_{0}^{t} \int_{\Omega}\left|\frac{\partial \bar{B}}{\partial \tau}\right|^{2} d x d \tau d t .
$$

From the Gronwall lemma, we finally obtain $\bar{B}=0$ and therefore, by comparison, $\bar{E}=\bar{H}=0$. Hence, all solutions coincide on $U_{r}$, independently on the way we constructed the sequence of data with compact support.

Outside $U_{r}$ the solution of (4.7) is given by the explicit formula for the solution of the linear wave equation. More precisely, outside $U_{r}$ (4.7) reduces to the following

$$
\left\{\begin{array}{l}
\frac{\partial E}{\partial t}+J_{\text {ext }}+\frac{\partial B}{\partial x}=0 \\
\frac{\partial B}{\partial t}+\frac{\partial E}{\partial x}=0
\end{array}\right.
$$

This means that

$$
\frac{\partial^{2} E}{\partial t^{2}}-\frac{\partial^{2} E}{\partial x^{2}}=-\frac{\partial J_{\text {ext }}}{\partial t}=: \tilde{J}
$$

and the initial conditions are

$$
E(x, 0)=E_{0}^{2} \quad \frac{\partial E}{\partial t}(x, 0)=-\frac{\partial B}{\partial x}(x, 0)-J_{\text {ext }}(x, 0)=: \tilde{E} .
$$


The solution for the linear wave equation can be represented as

$$
E(x, t)=\frac{1}{2}\left[E_{0}^{2}(x+t)-E_{0}^{2}(x-t)\right]+\frac{1}{2} \int_{x-t}^{x+t} \tilde{E}(\xi) d \xi+\frac{1}{2} \int_{0}^{t} \int_{x-t+\tau}^{x+t-\tau} \tilde{J}(\tau, \xi) d \xi d \tau,
$$

for $(x, t) \in[\mathbb{R} \times(0, T)] \backslash U_{r}$, and the assertion follows.

Acknowledgement. The authors wish to thank Gary Friedman for many useful discussions concerning the physical motivation of the model problem.

\section{References}

[1] H. Brezis: Analyse fonctionnelle, Théorie et applications, Masson, Paris (1983).

[2] M. Brokate, J. Sprekels: Hysteresis and phase transitions, Applied Mathematical Sciences, 121, Springer-Verlag, New York (1996).

[3] M. Brokate, A. Visintin: Properties of the Preisach model for hysteresis, J. Reine Angew. Math. 402 (1989) 1-40.

[4] R. Courant, D. Hilbert: Methoden der Mathematischen Physik, Vol. I, II, Interscience Publishers, Inc., N.Y., (1943).

[5] M. Eleuteri: Well posedness results for a class of partial differential equations with hysteresis arising in electromagnetism, Nonlinear Analysis, Real World Applications, to appear.

[6] J.D. Kraus, D.A. Fleisch: Electromagnetics, McGraw-Hill, Boston, (1999).

[7] M.A. Krasnosel'skiI, A.V. PokrovskiǏ: Periodic oscillations in systems with relay nonlinearities, Soviet Math. Dokl. 15 (1974) 873-877.

[8] M.A. Krasnosel'skiĬ, A.V. PokrovskiǏ: Modelling transducers with hysteresis by means of continuous systems of relays, Soviet Math. Dokl. 17 (1976) 447-451.

[9] M.A. Krasnosel'skIॅ, A.V. PokrovskiǏ: Systems with hysteresis, Springer, Berlin (1989), Russian edition: Nauka, Moscow (1983).

[10] P. KREJČÍ: On Maxwell equations with the Preisach hysteresis operator: the one-dimensional time-periodic case, Apl. Mat. 34 (1989) 364-374.

[11] P. KREJČí: Hysteresis, Convexity and Dissipation in Hyperbolic Equations. Gakuto Int. Ser. Math. Sci. Appl., Vol. 8, Gakkōtosho, Tokyo, 1996.

[12] P. KREJČÍ: Long-time behaviour of solutions to hyperbolic equations with hysteresis. Handbook of Evolution Equations: Evolutionary Equations, Vol. 2 (eds. C. M. Dafermos and E. Feireisl), Elsevier 2005, 303-370. 
[13] P. Krejčí, Ph. Laurençot: Generalized variational inequalities. J. Convex Anal. 9 (2002), 159-183.

[14] P. KRejČí, Ph. LaurenÇOt: Hysteresis filtering in the space of bounded measurable functions. Boll. Unione Mat. Ital. 5-B (2002), 755-772.

[15] E. Madelung: Über Magnetisierung durch schnellverlaufende Ströme und die Wirkungsweise des Rutherford-Marconischen Magnetdetektors. Ann. der Physik 17 (1905), 861-890 (In German).

[16] I.D. Mayergoyz: Mathematical models of hysteresis, Springer, New York (1991).

[17] F. Preisach: Über die magnetische Nachwirkung, Z. Physik, 94 (1935), 277302. (In German).

[18] A. Visintin: On the Preisach model for hysteresis, Nonliner Analysis, T.M.A. 9 (1984) 977-996.

[19] A. Visintin: Differential models of hysteresis, Springer, (1994). 